\title{
The spectral variability hypothesis does not hold across landscapes
}

\author{
Sebastian Schmidtlein* \& Fabian Ewald Fassnacht* \\ * Equal contribution
}

Family names in bold letters

Affiliation of both authors: Karlsruhe Institute of Technology (KIT), Institute of Geography and Geoecology, Kaiserstr. 12, 76131 Karlsruhe, Germany

Email addresses: schmidtlein@kit.edu, fabian.fassnacht@kit.edu

Corresponding author: Sebastian Schmidtlein

Keywords: Biodiversity, Floristic survey, Plant species richness, Spatial non-stationarity, SVH, Zoning effects

Color print is required for Figure 1 and Figure 4

\section{Abstract}

One of the biodiversity metrics to track from space is the spatial variability in reflectance that has previously been proposed as a proxy of species counts per unit area. The corresponding hypothesis is known as the Spectral Variability Hypothesis $(\mathrm{SVH})$. Little attention has been paid so far to the questions whether the SVH holds over broader regions and across time. Here, we addressed these questions by using a spatially contiguous dataset of vascular plant species occurrences in Southern Germany along with MODIS data at 14 time steps. The floristic dataset consists of species occurrence data for 815 areas of 10 longitudinal by 6 latitudinal minutes (approximately $12 \mathrm{~km}$ by $11 \mathrm{~km}$, referred to as mapping units). The spectral variability in space (within these units) was 
determined using MODIS pixels of $0.5 \mathrm{~km}$ by $0.5 \mathrm{~km}$. We used two different measures of spectral variability in combination with a moving window approach to derive statistical links between spectral variability and species counts through space and time. The moving windows consisting of several mapping units were shifted in space and meanwhile used as target areas for correlation analyses.

The performance of the spectral variability to predict species counts was influenced by the location and the extent of the reference windows. In some regions, high spectral variability was connected to high species counts. In other regions, comparably low spectral variability was linked to high species counts and vice versa. Furthermore, the relation between spectral variability and species varied with season. Certain areas changed from almost no correlation to very high correlation depending on the applied scene. Also, the applied spectral variability measure had a notable influence on the observed results.

Based on these results, we conclude that the spectral variability hypothesis does not hold across landscapes at this spatial grain. Using spectral variability alone as a proxy for species counts in a monitoring approach for larger extents and grains is therefore unlikely to work in many parts of the world. This does not mean that it cannot help as a covariate in analyses with more predictors.

\section{Introduction}

An increased awareness of the need for biodiversity conversation led to international agreements about sustaining biodiversity for future generations. This includes the need for an effective monitoring and early warning system. One candidate technique for such kind of a system is remote sensing. A set of "biodiversity metrics to track from space" has been recently proposed by the GEO BON initiative (Skidmore et al. 2015). These metrics, many related to ecosystem structure and functioning, can be linked to biodiversity and are at the same time detectable with remote sensing. However, the list of metrics does not include the number of species per unit area, shortly called species density or species count, as the most common field-based measure of biodiversity. Species 
density is missing because there is no obvious physical link to reflected electromagnetic radiation, which is the base for remote sensing.

Attempts that have been made so far to map plant species counts are of mostly correlative nature and rely on the observation that visible properties of the earth surface are in one or another way linked to species numbers. While these links sometimes allow good local estimates, the approach is hardly transferable in space and time and depends on calibration on the spot. The correlative approach was found to work in all major domains of remote sensing like multispectral remote sensing (e.g., Foody \& Cutler 2003, 2006, Feilhauer \& Schmidtlein 2009, Rocchini 2007), hyperspectral remote sensing (e.g., Laurin et al. 2014) as well as active remote sensing or combinations (e.g., Camathias et al. 2013, Higgins et al. 2014, Lopatin et al. 2015, 2016, Simonson et al. 2012, Hernández-Stefanoni et al. 2014). Reviews can be found for example in Wang et al. (2010) and Rocchini et al. (2010).

A more generic way is to relate plant species counts to variation of reflectance. A hypothesis called the "Spectral Variability Hypothesis" (SVH) has been formulated by Palmer et al. $(2000,2002)$ and suggests that variability in reflectance or "spectral variability" of an area is an expression of spatial ecosystem heterogeneity and therefore related to plant diversity. If plant individuals are large or pixels are small, variability in reflectance may also be a direct expression of varying species reflectances (Carlson et al. 2007). We use the term "spectral variability" instead of the often used "spectral variation" because the former one is the one coined in the original publication by Palmer (2000).

Table 1 summarizes studies that addressed the spectral variability hypothesis (compare also studies listed in Rocchini et al. 2010). In most of the studies the spectral variability hypothesis was tested with datasets covering relatively small spatial extents (400 ha - several tens of sq. $\mathrm{km}$ ), with the studies of Oindo and Skidmore (2002), covering the whole of Kenya, and Rocchini et al (2014) covering North America being exceptions. In most studies, the spatial grain (Dungan et al. 2002) of 
the remote sensing data was fine with pixel sizes of 1-30 m.

Field data was often limited with most studies applying less than 100 field samples. The spectral variability measures differed amongst the studies and ranged from relatively simple measures such as the coefficient of variation of digital numbers or reflectance values of single bands to measures obtained from multi-dimensional feature spaces incorporating several spectral bands simultaneously (e.g., mean Euclidean distance, mean distance from centroid or the interquartile range divided by the median). Reconciling the original idea of the SVH, a further suitable measure for spectral variability that relates to spatial ecosystem heterogeneity or species counts could base on an unsupervised classification with a high number of classes. Clustering the given remote sensing image into a high number of discrete classes should result in an abstract representation of the environmental heterogeneity. If the SVH holds, a high number of classes occurring at a small spatial extent should then indicate a high environmental heterogeneity and therefore relate to high species numbers. At fine spatial grain, more classes per unit area could be a direct surrogate of species counts (Féret \& Asner 2014).

In many of the studies summarized in Table 1, low to moderate correlations between field measured biodiversity measures and spectral variability measures were found (e.g., Hall et al. 2010, 2012, Rocchini et al. 2004) but the variety of results is conspicuous and due to the differences in study designs results can hardly be interpreted further.

Table 1: Overview of remote-sensing based studies related to the spectral variability hypothesis. [see separate file]

Reasons leading to these varying results may be connected to spatial grain effects which can be subdivided into (a) effects related to the pixel size of the applied remote sensing data and (b) effects originating from the size of the field plots. Furthermore, there may be effects of (c) extent, zoning or selection of the areas of investigation (Dungan et al. 2002, Rocchini et al. 2010). Other potential sources of differences are (d) spectral resolution, and (e) the timing of the investigation since 
canopy reflectance changes with season, due to leaf phenology or ephemeral plants.

\section{Effects of pixel size and spectral resolution}

Rocchini (2007) investigated effects of pixel size but found ambiguous results as both the very fine grain Quickbird data (2.4 $\mathrm{m}$ pixel size) and the Landsat data (30 $\mathrm{m}$ pixel size) produced high correlations with plant species counts while ASTER data (15 m pixel size) showed notably reduced correlations. The authors argue that intermingling effects of differing spectral resolutions may be one reason for these inconclusive results. A theoretical discussion on the ideal pixel size for biodiversity estimation studies is given in Rocchini et al. (2010). An increased spectral resolution was found to be "beneficial" (in terms of increased coefficients of correlation between spectral variability and species numbers) in several studies (e.g., Rocchini 2007, Oldeland et al. 2010, Nagendra et al. 2010).

\section{Effects of the size of field reference sites}

Rocchini et al. 2014 reported an increasing spectral variability with increasing size of mapping units with areas ranging from a few to ca. $48,000 \mathrm{~km}^{2}$. The effect of field plot or mapping unit size on the correlation between spectral variability and species counts was investigated by Rocchini et al. (2004) who found higher correlations when going from $100 \mathrm{~m}^{2}$ plots to nested macro-plots with a size of 1 ha. The same was reported by Oldeland et al. (2010) for plot sizes of $100 \mathrm{~m}^{2}$ and $1000 \mathrm{~m}^{2}$ and Viedma et al. (2012) when going from $1 \mathrm{~m}^{2}$ to $100 \mathrm{~m}^{2}$. Oldeland et al. (2010) give a more detailed discussion on this issue and mention several more studies, backing up the assumption that increased field plot size will lead to an improved performance of spectral variability as a proxy of species density. Furthermore, the authors mention that also the shape of the plot may influence the results, as "elongated plots typically capture a wider range of ecological gradients" (Oldeland et al. 2010, p. 394). This points to an important variable contributing to the observed correlations: Beta diversity as a pre-requisite for heterogeneity of reflectance and higher numbers of species. With no beta-diversity, low spectral variability may be related to any species counts, at least as long 
individual plants do not exceed the subpixel level (Schmidtlein and Sassin 2004). A concrete example could be the comparison between homogeneously managed, species-rich and species-poor grasslands. In most remote sensing datasets, both ecosystems would show a comparably small spectral variability with one ecosystem being species rich and one being species-poor.

\section{Effects of Zoning and Seasonality}

It is quite obvious that differences in outcomes of the studies listed above may be due to the individuality of the investigated patterns, like the characteristics of ecosystems and their spatial arrangement. For broader areas, relations between spectral variability and plant species counts have been investigated by Rocchini et al. (2014), with the result of no clear signal. However, no study so far has checked whether this lack of signal is due to contradictory signals in subsets of areas (that is, both positive and negative correlations between spectral variability and plant species). Just like spatial non-stationarity, the temporal component is under-examined. It is likely that the correlation between species counts and spectral variability varies over the course of a year. This variation is presumably related to a multitude of processes including for example temporal differences amongst species in leaf green-up in the spring, leaf-area development over the year, land-use like mowing and grazing, flowering periods, leaf senescence in fall or fires. One study touched this topic so far and found strongest correlations between NDVI and species counts in a Landsat image from May when comparing 5 different points in time during the vegetation period (Levin et al. 2007).

We have thus shown that there is a number of aspects of the spectral variability hypothesis that has not yet been investigated thoroughly. In this study we address four knowledge gaps by focusing on the following four research questions:

1. Does the spectral variability hypothesis hold over a larger extent $\left(106.300 \mathrm{~km}^{2}\right)$ in Germany?

2. How is the performance of spectral variability as a proxy of plant species numbers influenced by the location and the extent of the reference region?

3. Is the relation between spectral variability and plant species counts stable over the seasons? 
4. Does the measure of spectral variability affect the results?

To answer these questions we made use of a dataset containing field-collected, spatially-continuous counts of plant species counts throughout the German federal states of Bavaria and BadenWuerttemberg. This reference was related to a series of 8-day MODIS mosaics.

\section{Methods and materials}

\subsection{Study site}

The studied area embraces the two German federal states Bavaria and Baden-Wurttemberg with a total area of approximately $106.300 \mathrm{~km}^{2}$. The two states cover a wide range of geological and climatic conditions which translate into 22 bio-geographic regions (of third order according to the German system of eco-regions, (Meynen \& Schmithüsen 1953-1962) with diverse hydrological and ecological properties. The altitudinal range varies between $85 \mathrm{~m}$ above sea level (Rhine river close to Mannheim in Baden-Wuerttemberg) to $2962 \mathrm{~m}$ above sea level (the peak of the Zugspitze summit in the Bavarian Alps). Both states have a similar total forest cover of $38 \%$ and $37 \%$ for BadenWuerttemberg and Bavaria, respectively.

\subsection{Biodiversity dataset}

The biodiversity dataset applied in this study is a result of the Floristic Survey of Central Europe (Netzwerk Phytodiversität Deutschland \& Bundesamt für Naturschutz 2013). We used a subset of the German "FlorKart" database provided by the German Federal Agency of Nature Conservation (doi:10.15468/wnkii7). This dataset provides comprehensive lists of vascular plant species for grid cells of 10 longitudinal by 6 latitudinal minutes (approximately $12 \mathrm{~km}$ by $11 \mathrm{~km}$ ) (corresponding to ordinance survey maps of Germany with a scale of 1:25.000) or for quarters of these cells. We used entire grid cells for the sake of data consistency. Most of the mapping efforts took place between 1980 and 1990 but latest entries were completed only in 2000. The mapping was conducted by volunteering experts and contains 9317 plant species with taxa information until the sub-species level 
for the whole of Germany (Mahecha \& Schmidtlein, 2008). We considered taxon densities at the species level to increase comparability across the area.

We limited our research to 815 mapping units located in the federal states of Baden-Wuerttemberg and Bavaria as it has been shown that the floristic surveying methodology in these two states was very similar and a high consistency in the data can be expected (Mahecha \& Schmidtlein, 2008). A map depicting the vascular plant species counts for each mapping unit of the two states is given in Figure 1.

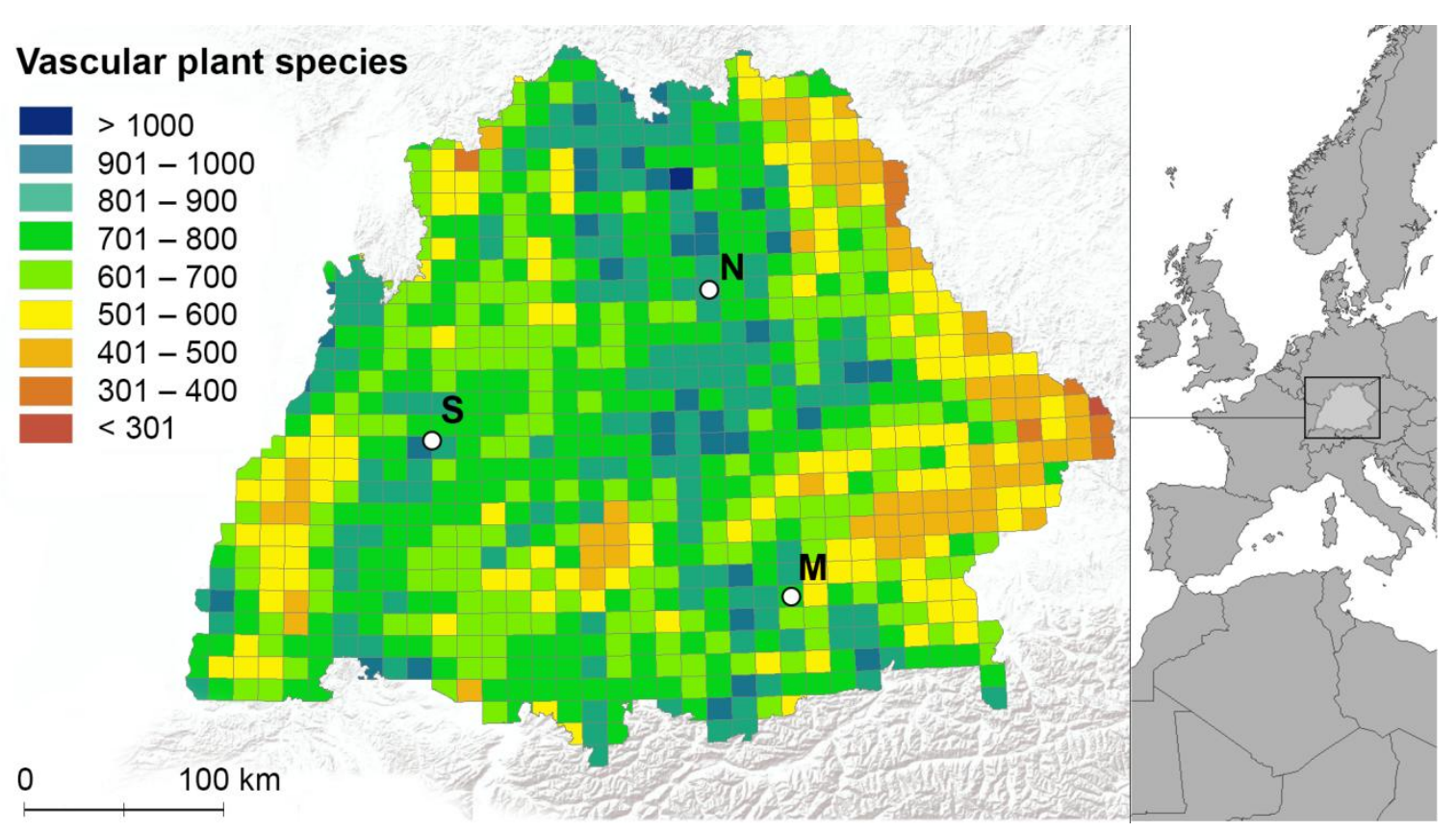

Fig. 1: Vascular plant species counts across the study area according to the "FlorKart" database. M $=$ Munich, $\mathrm{N}=$ Nuremberg, $\mathrm{S}=$ Stuttgart.

\subsection{Remote sensing data}

The spectral information used in this study was obtained from the Moderate Resolution Imaging Spectroradiometer (MODIS) sensor on board of NASA's Terra platform (Salomonson et al. 1989). We restricted the scenes to data obtained by the MODIS sensor on Terra to avoid spectral differences due to the differing overflight times of the MODIS sensors on Terra and Aqua. 
We downloaded 8-day reflectance composites (MODIS product MODO9A1), which are routinely created based on all data gathered by either of the MODIS sensors. These composites are artificial datasets composed of the "best" pixels of all scenes collected during an 8 day period. By compositing only the "best" pixels, problems related to high view-angles, aerosols and clouds are drastically reduced (LP DAAC). We restricted our data to scenes from 2001 to 2003 (the earliest available dates, closest to the reference dataset) and acquired only scenes that showed minimal remaining cloud-cover in the composites. The composites have a pixel size of $500 \mathrm{~m}$ and deliver spectral information in 7 bands (Table 2).

Table 2: MODIS bands of the MODO9A1 product

\begin{tabular}{llll} 
Band & Wavelength $[\mathbf{n m}]$ & Color & Information \\
\hline 1 & $620-670$ & Red & Land-cover change, Chlorophyll \\
2 & $841-876$ & Near infrared & Clouds, land-cover change \\
3 & $459-479$ & Blue & Separation between soil and vegetation \\
4 & $545-565$ & Green & Green vegetation \\
5 & $1230-1250$ & Near infrared & Canopy information \\
6 & $1628-1652$ & Shortwave & Separation between clouds and snow \\
7 & $2105-2155$ & Shortwave & Cloud and land properties \\
\hline
\end{tabular}

We finally selected 14 scenes with a good seasonal coverage. Acquisition dates are listed in Table 3. All scenes were stacked and then georeferenced to a RGB-Landsat composite of the study area (UTM zone $32 \mathrm{~N}$ ). Based on a visual assessment this procedure resulted in an accuracy of approximately 1-2 MODIS pixels, which is adequate for the scale of the study..

Table 3: Acquisition dates of all MODIS composites used in the study

\begin{tabular}{llllll} 
Timestep & Day & Day & Year & Visible snow & Comment \\
\hline 1 & 10.02 .2001 & 041 & 2001 & Yes \\
2 & 06.03 .2002 & 065 & 2002 & Yes \\
3 & 22.03 .2002 & 081 & 2002 & Yes
\end{tabular}




\begin{tabular}{llllll}
4 & 30.03 .2002 & 089 & 2002 & Yes & \\
5 & 15.04 .2003 & 105 & 2003 & Yes & \\
6 & 17.05 .2001 & 137 & 2001 & Yes & \\
7 & 18.06 .2003 & 169 & 2003 & Yes & Few bright outlier pixels \\
8 & 28.07 .2001 & 209 & 2001 & No & Few bright outlier pixels \\
9 & 05.08 .2003 & 217 & 2003 & Yes & \\
10 & 06.09 .2002 & 249 & 2002 & No & \\
11 & 14.09 .2003 & 257 & 2003 & Yes & \\
12 & 22.09 .2003 & 265 & 2003 & Yes & \\
13 & 16.10 .2003 & 289 & 2003 & Yes & Few bright outlier pixels \\
14 & 01.11 .2003 & 305 & 2003 & Yes & Presumably some snow-covered \\
\hline
\end{tabular}

Outlier pixels having a reflectance value over $25 \%$ in the red channel were masked to exclude pixels affected by artefacts or snow. Second, to allow for a balanced contribution of each spectral band to the spectral variability measure, we standardized the reflectance values of each individual band with the function "scale" of the R-package "raster" (Hijmans, 2012). This resulted in a stack of raster layers, where each layer featured values between 0 and 1 and the standard deviation of all pixels equaled 1 . With this standardization, all covered spectral domains contributed equally to the measures of spectral variability instead of giving emphasis on, for example, the NIR signal. Apart from the mentioned outlier pixels we did not mask any part of the area meaning that reflectance of all landcover types entered the analysis.

\subsection{Calculation of spectral variability measures}

Each pixel of the MODIS dataset can be pictured as a point in a 7-dimensional feature space with the 7 dimensions representing the 7 spectral bands. If all 730 pixels (median) falling into one field mapping unit (10 longitudinal by 6 latitudinal minutes, approximately $12 \mathrm{~km}$ by $11 \mathrm{~km}$ ) are depicted in this feature space, it is possible to calculate, for example, the mean Euclidean distance between the points or alternatively the mean distance to the "spectral" centroid of the dataset (Rocchini et al. 2007). These measures summarize the spectral variability of all pixels in a single value. Both measures are highly correlated and we therefore decided to continue with the simpler and 
more intuitive Euclidean distance. To address potential, remaining outliers, we only used pixels falling between the $2 \%-98 \%$ percentiles. The Euclidean distance was calculated as spectral variability measure for each of the MODIS time steps (compare Table 3) within each of the field sample plots.

As a second measure for spectral variability we calculated the number of classes of an unsupervised k-means classification in each mapping unit. The k-means classification was conducted separately for each of the 14 MODIS scenes and the number of clusters was arbitrarily set to 100 . We hypothesized that this measure might be better related to environmental heterogeneity than the conventionally used mean Euclidean distance which "rewards" dissimilarity between classes while disregarding the variety. That is, two spectrally very different classes (like forests and meadows) have a high Euclidean distance with their co-occurrence leading to high spectral variability values despite only two classes are present. More classes would not necessarily increase the Euclidean distance but are more likely linked to more habitats and therefore more species. Counting clusters should be more related to counting habitats and therefore to counting species.

\subsection{Area-wide correlation between spectral variability and species counts}

We calculated Spearman rank correlations between spectral variability in individual mapping units and the respective species counts. This was done for both spectral variability measures (mean Euclidean distance and k-means based number of clusters) and for all 14 time-steps. To take account of the presence of spatial autocorrelation we calculated the effective sample size and the resulting degrees of freedom using Dutilleul's method (Dutilleul 1993).

\subsection{Moving window approach}

Motivated by the spatial continuity of our reference dataset, we decided to not only validate the spectral variability hypothesis over the whole study site but also over spatial subsets of it. We therefore implemented a moving window approach in which a circular window including multiple map- 
ping units runs over the study area and derives the correlation between spectral variability measures and species counts. This approach takes also account of the presence of spatial autocorrelation in the data. As correlation measure we used Spearman's rank correlation coefficient to account for non-linearity. As in the global model, we corrected the significance values with Dutilleul's method (Dutilleul 1993). The moving window approach was implemented with the "spdep" package of R (Bivand and Piras, 2015).

In each step, the window was moved by one mapping unit (10 longitudinal minutes or 6 latitudinal minutes). To define the analysis window around a central mapping unit, all neighboring units were included that fell within a distance including $2 \times r$ units in North-South or East-West direction. To investigate the effect of the study area size on the relationship between spectral variability and species counts, the moving window approach was run for window sizes of $r=3-10$. For the mapping units included in the analysis windows, the Spearman rank correlation between spectral variability and species counts was calculated. For a visualization and spatial interpretation, the result for each window was attributed to its central mapping unit. The procedure was repeated for each unit and its corresponding window.

\section{Results}

\subsection{Relation between species counts and spectral variability when taking into account the complete area}

There was no clear relation between species counts and spectral variability when taking into account the complete area ((Fig. 2). Irrespective timing, rho values for the complete area were usually positive, with a maximum of rho $=0.36$ with the kmeans-based measure of spectral variability. Some of the higher rho values were significant at $\mathrm{p}<=0.001$ after correcting for spatial autocorrelation (without correction, most values resulted significant at this level). The correlations with Euclidean-based values were weak with a maximum of rho $=0.23$ and never reached significance at $\mathrm{p}<=0.001$.The scatterplots in Fig. 2 show situations for the MODIS timesteps with 
highest rho values.

Table 4: Spearman rank correlations between species counts and spectral variability (calculated as mean Eucliden distance and number of k-means classes) in all mapping units on 14 dates, using a window size of $r=3$ mapping units. This smaller window size gave slightly higher absolute rho values than larger windows. The reported p-values are corrected for spatial autocorrelation.

\begin{tabular}{ccccc} 
Timestep & $\begin{array}{c}\text { Euclidean distance based } \\
\text { rho }\end{array}$ & $\mathbf{P}$ & $\begin{array}{c}\text { k-means based } \\
\text { rho }\end{array}$ & $\mathbf{P}$ \\
\hline 1 & 0.003 & 0.976 & 0.252 & 0.000 \\
2 & 0.027 & 0.793 & 0.205 & 0.017 \\
3 & 0.106 & 0.244 & 0.241 & 0.012 \\
4 & -0.059 & 0.553 & 0.195 & 0.023 \\
5 & -0.062 & 0.575 & 0.364 & 0.000 \\
6 & 0.043 & 0.639 & 0.228 & 0.006 \\
7 & 0.066 & 0.496 & 0.342 & 0.000 \\
8 & 0.228 & 0.010 & 0.326 & 0.000 \\
9 & 0.119 & 0.156 & 0.224 & 0.001 \\
10 & 0.065 & 0.490 & 0.342 & 0.000 \\
11 & 0.203 & 0.007 & 0.167 & 0.060 \\
12 & 0.077 & 0.323 & 0.204 & 0.004 \\
13 & 0.145 & 0.094 & 0.224 & 0.002 \\
14 & -0.075 & 0.496 & 0.300 & 0.000
\end{tabular}

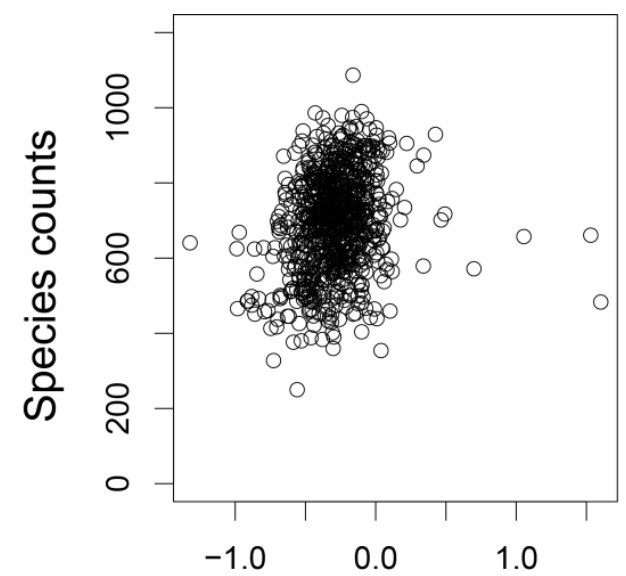

Spectral Var. (log mean Eucl. dist.)

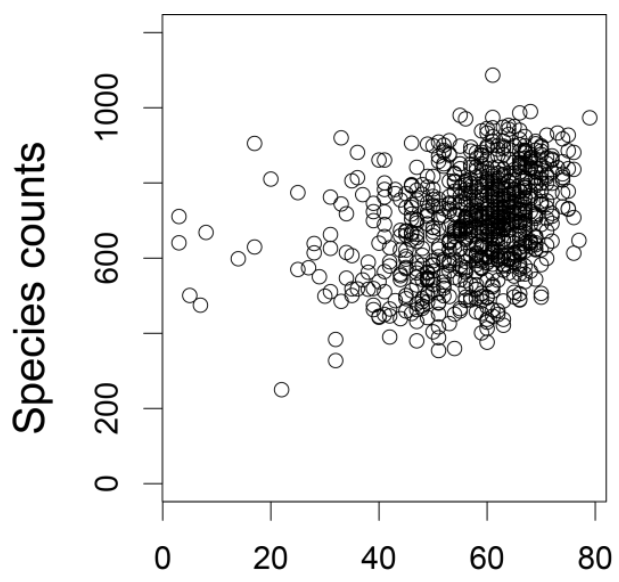

Spectral Var. (k-means)

Fig. 2: Overall relation between species counts and spectral variability expressed as logarithmic mean Euclidean distance (left panel) and number of k-means classes in a mapping unit (right panel). 
Points are individual mapping units. These are results for timesteps 8 and 5 and window size $r=3$, featuring the highest correlations in terms of Spearman rho.

\subsection{How is the link between spectral variability and species counts influenced by the season, position and extent of the reference region?}

When reducing the reference area to spatial subsets using the moving windows approach, we observed clear but contrasting patterns of correlation between spectral variability and species counts across time and space. The moving windows approach provided evidence of spatial and temporal non-stationarity in the relationship between species counts and spectral variability. In the following, we first report seasonal differences, then the effects of position and size of the investigated area.

\subsubsection{Effects of season}

In the results for the moving window approach, seasonal differences led to an ephemeral appearance of areas with high correlations between species counts and spectral variability. While wide areas showed quite, but not exactly, consistent correlations across seasons (like the Northern Black Forest, labeled with [1] in Fig. 3), other areas fluctuated between positive and negative signs (like the Upper Palatinate Forest, labeled with [2] in Fig. 3). This variability with season is apparent for both examined spectral variability measures. Significance values for the examples from Fig. 3 are provided in the supplementary materials S1. High absolute rho values were usually significant at $\mathrm{p}<=0.01$. 

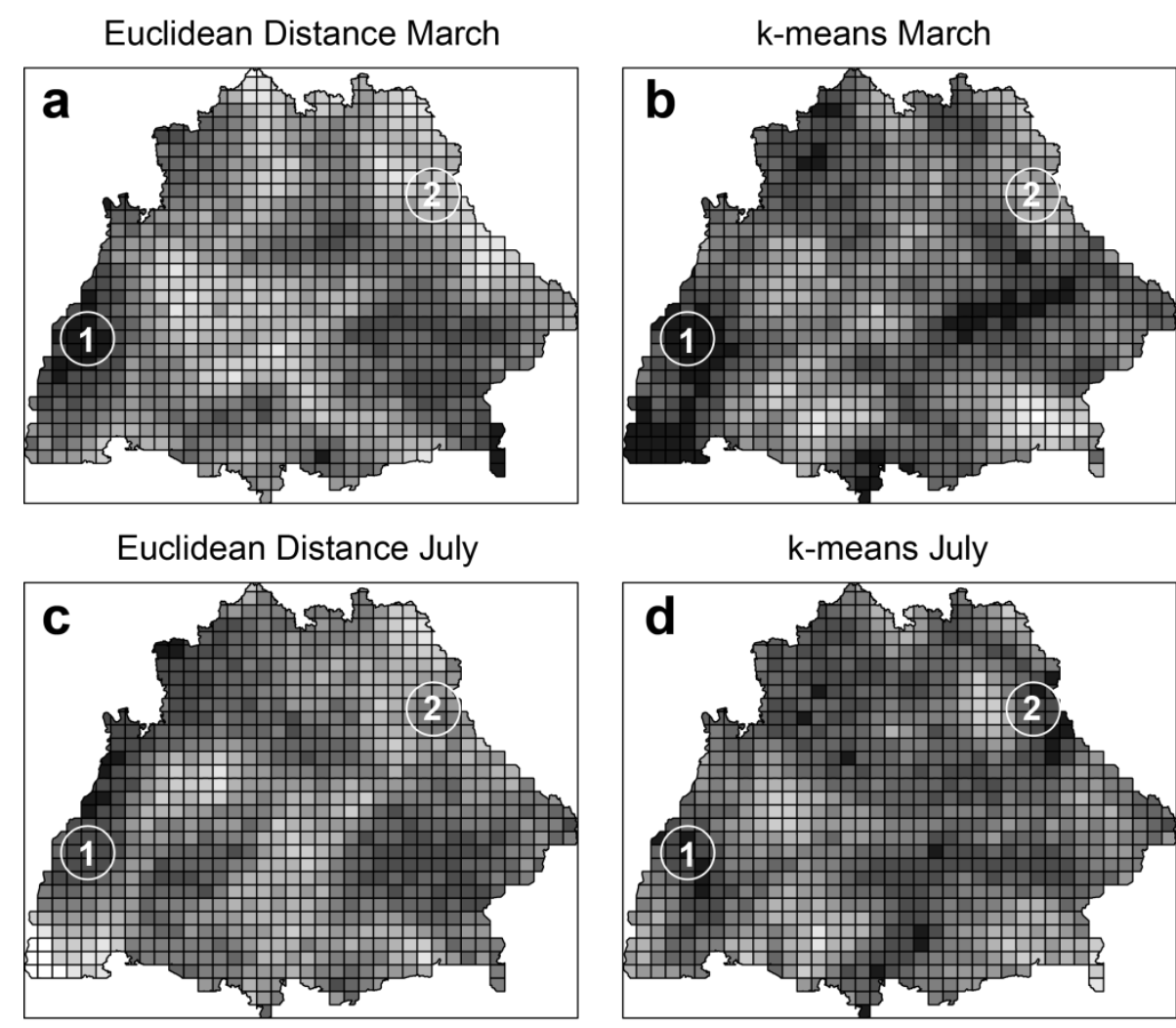

\section{Euclidean Distance October}
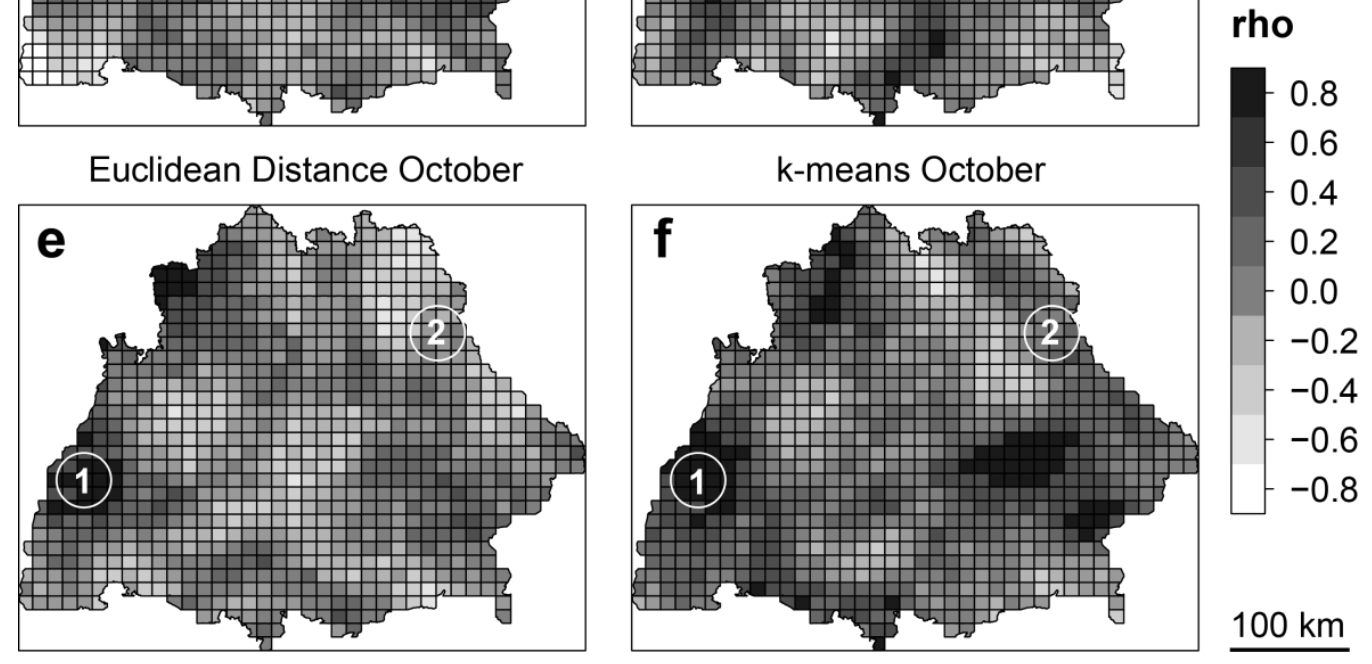

Fig. 3: Spearman rank correlation between species counts and spectral variability across space and seasons. The values were calculated using the moving-window approach with mean Euclidean distances (left column) and number of k-means classes (right column) as spectral variability measures. In all cases the radius $r$ of the moving window was 3 mapping units. $1=$ Northern Black Forest, 2 = Upper Palatinate Forest.

\subsubsection{Effects of position and size of the investigated area}

We found peaks of correlation in areas with gradients between relatively species-poor and speciesrich landscapes. These gradients usually coincide with distinct differences in landscapes (for example the species-poor Black Forest with forested hills vs. species-rich, more patchy landscapes in lowlands). Both measures of spectral variability led to similar patterns in parts of the area but we 
also found notable differences. For example, we observed a strong positive correlation between species counts and spectral variability with k-means in the most south-western part of the study area (Southern Black Forest) that contrasts with negative correlations obtained using Euclidean distances (Fig. 3).

A closer look on these results reveals that patchiness within floristic mapping units in contrast to homogeneity created strong gradients in spectral variability across mapping units. If these gradients matched up with a gradient in species counts, we observed high correlations. However, the direction of the correlation could be in both directions depending on which of the landscapes (the heterogeneous or the homogeneous) were species-rich.

An example for, at the given spatial grain, spectrally homogeneous landscapes with low species counts neighboring spectrally heterogeneous landscapes with high species counts is the transition between the largely forested Black Forest region and the adjacent, patchier Albvorland-Gäue area. Fig. 4 shows the distribution of species counts (4 b), spectral variability (4 c), and corresponding correlations (4 a). In this figure, the Black Forest is marked with [1] and the Albvorland-Gäue region is labeled with [2]. Fig. 5 (sub-graphs 1 and 2) shows illustrative examples for both landscapes. The Black Forest is poor in species and poor in spectral classes while the Albgäu is richer in species and richer in spectral classes. The matching gradients between both areas result in strong positive correlations between species counts and spectral variability. Negative correlations are for example found along a gradient between lower and higher pre-alpine landscapes, which is accompanied by a homogenization in agricultural land-use (a variety of crops in the lower parts, [3] in fig.4 and fig. 5, in contrast to grasslands in the higher parts [4] in fig. 4 and fig. 5). The spectrally more homogeneous grassland area features higher species counts. 


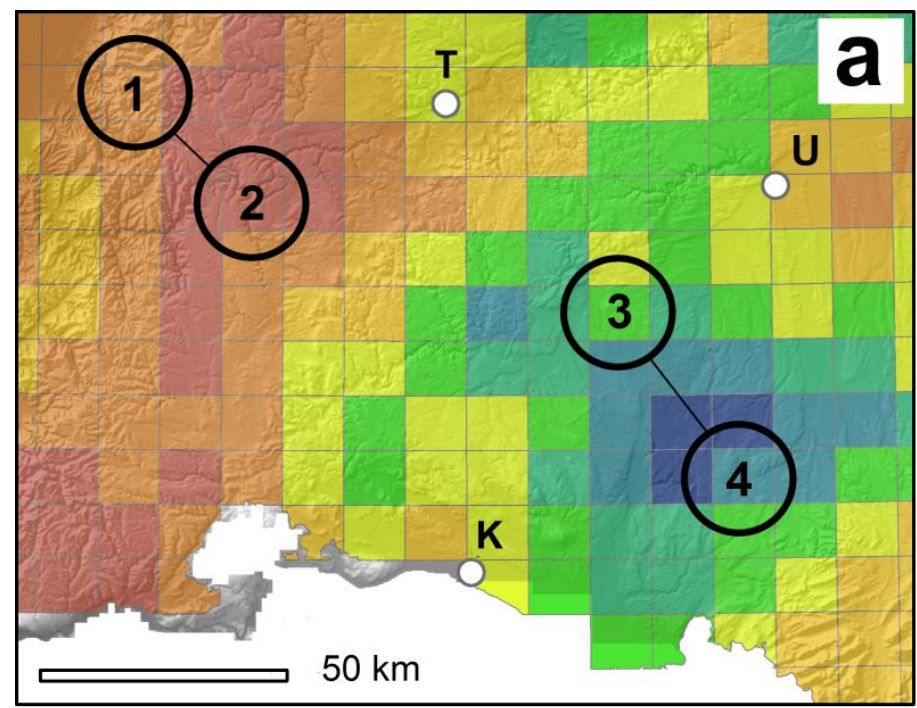

\section{rho}
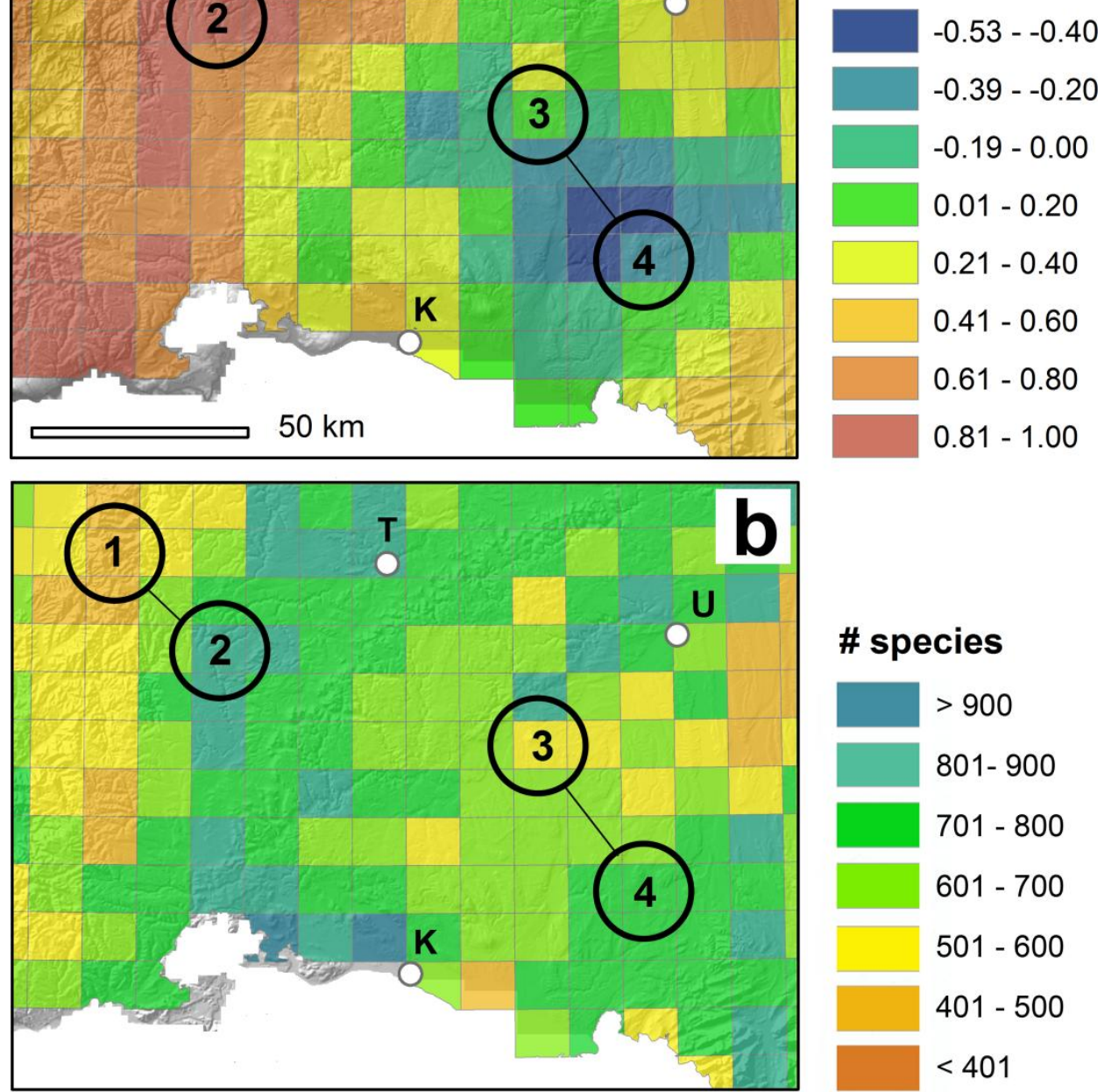

\section{\# species}
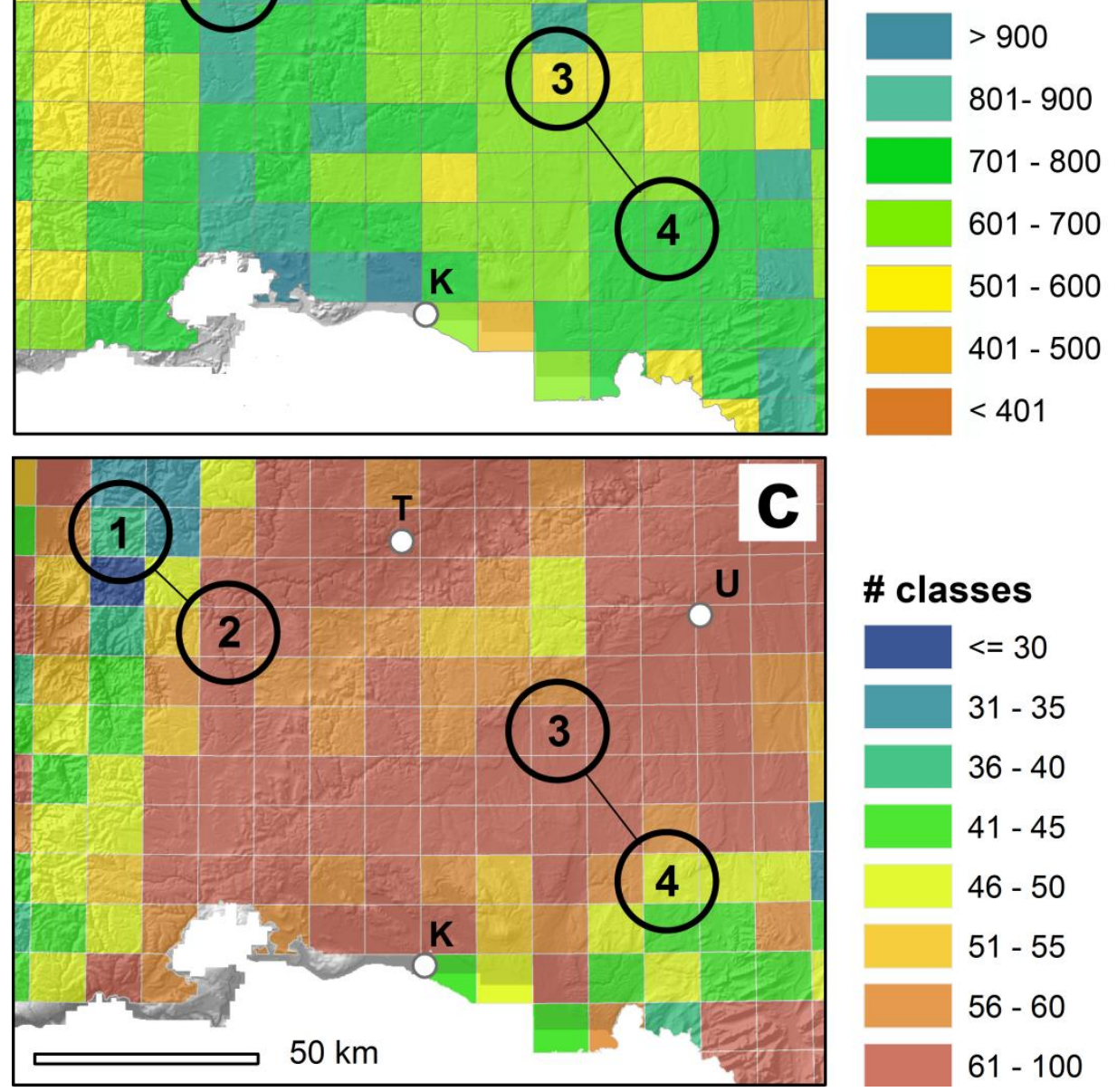

Fig. 4: Subset of the study area showing positive (a: labels 1 and 2) and negative correlations (a: labels 3 and 4) between species counts (b) and spectral variability (c). Moving windows-size: $r=3$ mapping units. Best k-means-based results from April are displayed. $\mathrm{K}=$ Constance, $\mathrm{T}=$ Tübingen, 
$\mathrm{U}=\mathrm{Ulm}$.

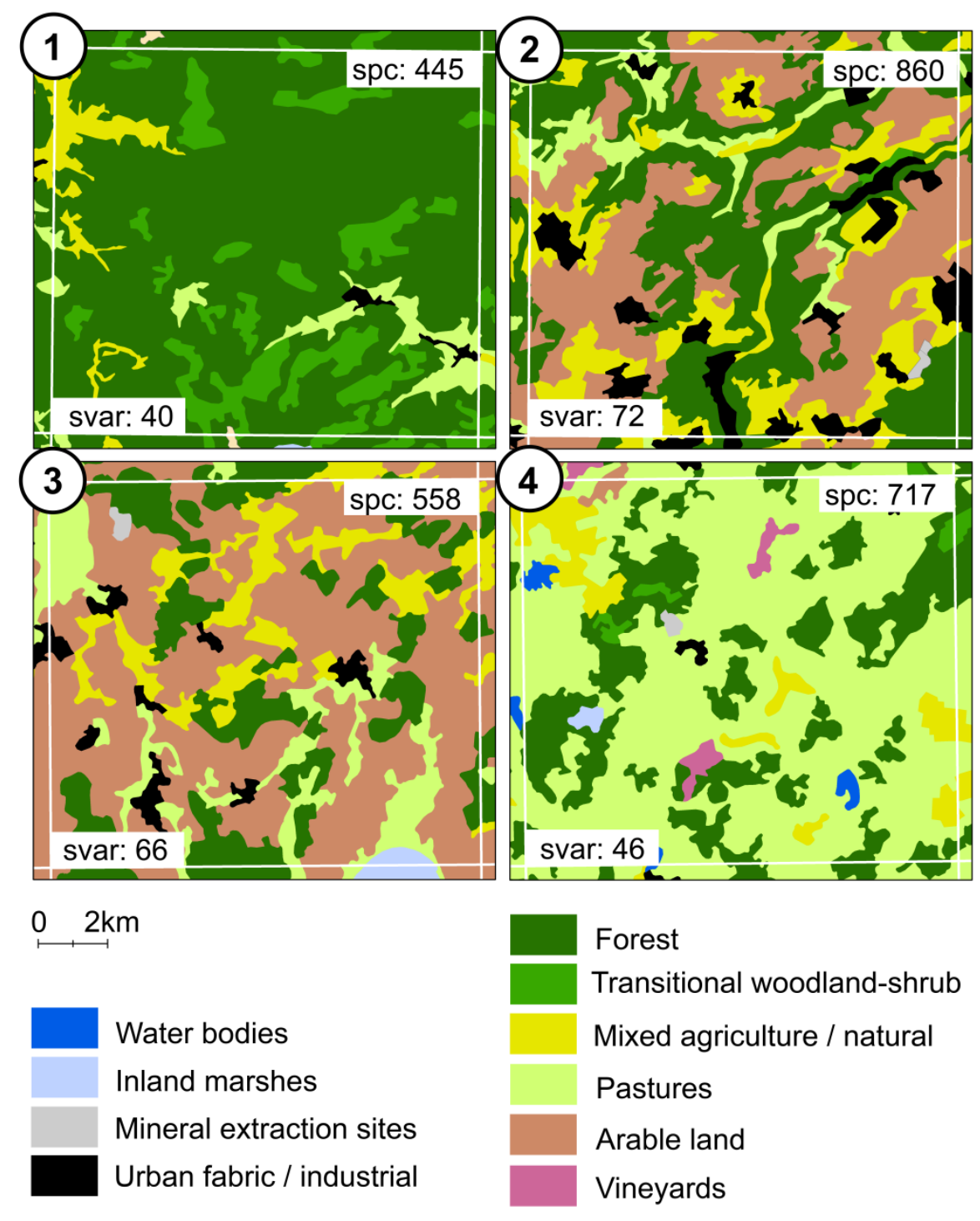

Fig. 5: Illustrative examples showing pairs of mapping units in areas with positive correlation between spectral variability and species counts (1 and 2) and in areas with negative correlation (3 and 4$) . \mathrm{spc}=$ vascuslar plant species counts, svar = spectral variability expressed as number of $\mathrm{k}-$ means clusters. The locations of the four mapping units is shown in Fig. 4 (numbers 1 to 4 ).

Reference data as in Fig. 4. The landcover classes are derived from Corine landcover information (Bossard et al. 2000). 
We modified the extent of the moving window in order to assess the influence of the size of the investigated area on the results (Fig. 6 shows the resulting patterns in correlation for $r=3$ to 5 mapping units). The maximum observed correlations between spectral variability and species counts were generally higher with smaller window sizes. With increasingly larger window sizes, areas of high correlations began to expand while maximum correlations decreased. We refrain from showing and interpreting results with $r>5$ because edge effects started to dominate the pattern.

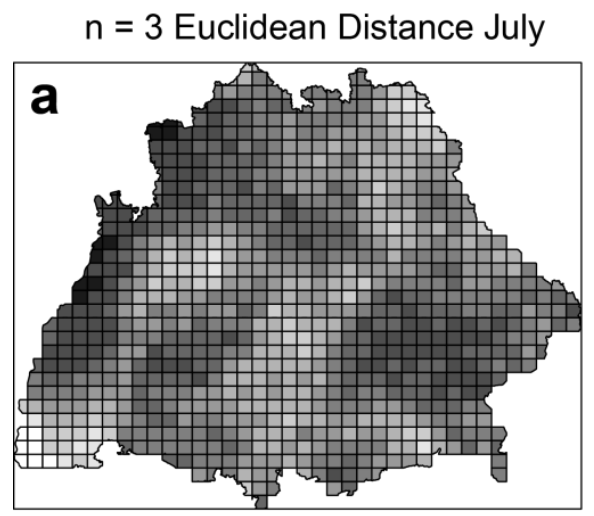

$\mathrm{n}=4$ Euclidean Distance July

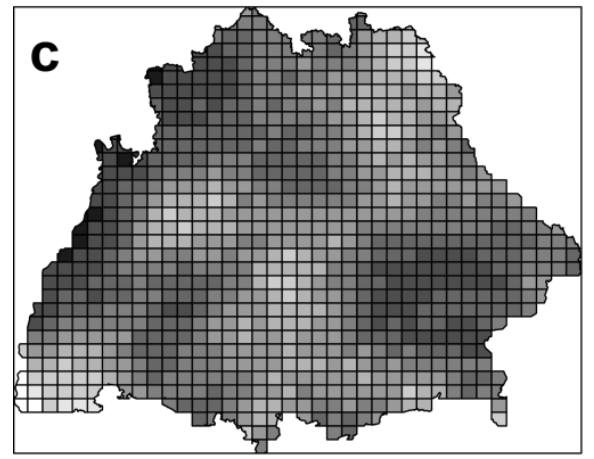

$\mathrm{n}=5$ Euclidean Distance July

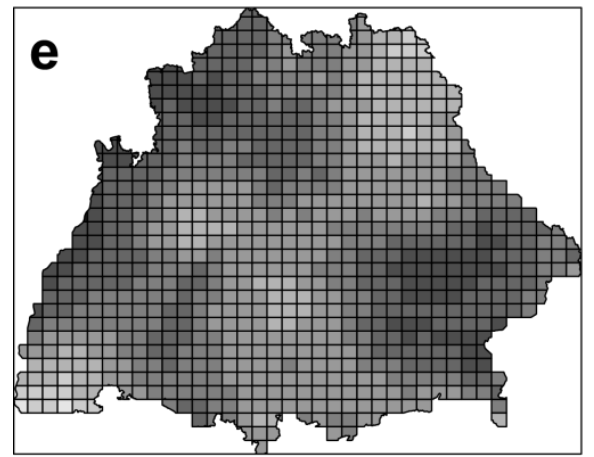

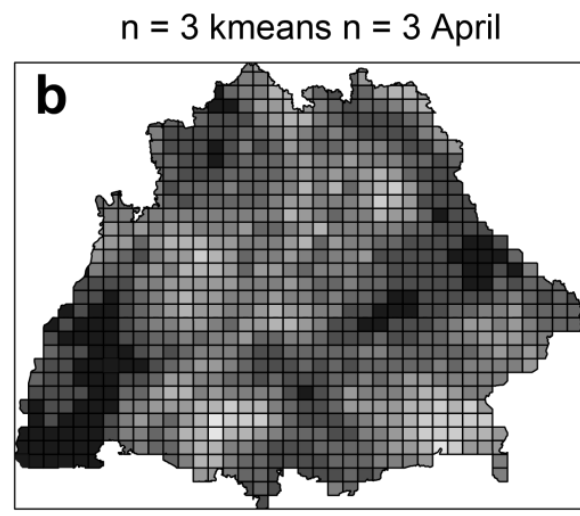

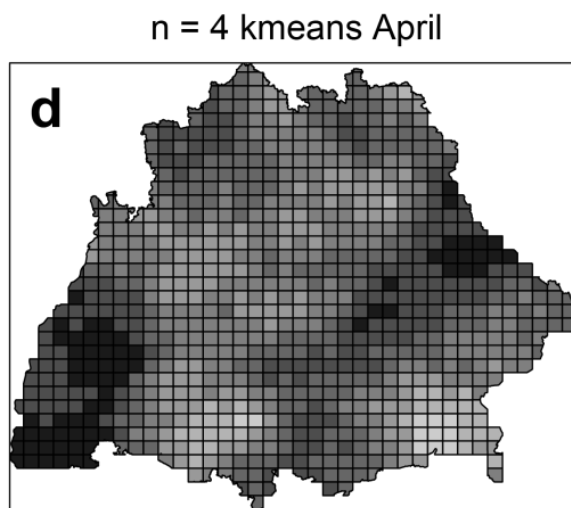

rho

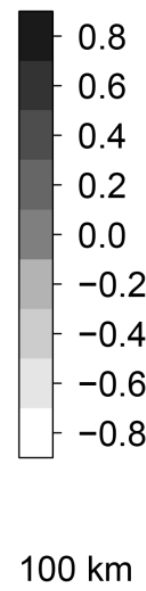

Fig. 6: Development of the correlation patterns with increasing size of the moving window. 
Displayed are the best results for the mean Euclidean distance (left panels) and the k-means-based measure of spectral variability (right panels) with moving window $r=3,4$ and 5 mapping units.

\section{Discussion}

This study combined a spatially contiguous reference set of species count values from southern Germany with 14 MODIS datasets of the same area to evaluate the spectral variability hypothesis $(\mathrm{SVH})$ at a coarse spatial grain (the species records relate to areas of approximately $12 \mathrm{~km}$ by $11 \mathrm{~km}$ and the spectral variability within these units was determined using pixels of $0.5 \mathrm{~km}$ by $0.5 \mathrm{~km}$ ). Due to the exceptional spatial consistency of the species dataset, we were able to investigate effects of study area size as well as study area location. The temporal discrepancy between species records and MODIS data (the former collected between 1980 and 2000, the latter 2001-2003), is not considered an issue. In Germany changes in landscape patterns and habitats do not take place at that speed, or only in rare and local exceptions.

Furthermore, we tested two conceptionally different spectral variability measures: the mean Euclidean distance between pixels in a mapping unit and the number of reflectance-based k-means classes in such a unit. In addition we also tested a spectral-temporal variability measure assuming that the temporal variability of the spectral signature observed over the course of a year may also contain relevant information related to species counts. However, the results for the spectraltemporal variability were worse than the results for spectral variability alone and we refrained from including these results in the current paper.

Our investigations clearly revealed problems in the concept of the SVH at the examined spatial grain and in the examined area. This applies to all investigated effects. In the following, we separately discuss the results concerning seasonality, study area size and location and end the discussion with thoughts on spatial grain and appropriate biodiversity measures. 


\subsection{Seasonality}

When comparing the correlation between spectral variability and species counts over the course of a year we observed strong variability. In some regions the observed correlation for differing months varied between almost no correlation up to very high values (Spearman rho $>0.7$ ). In some parts of the study area we observed changes from negative to positive correlations. This agrees well with ecological knowledge since phenology notably affects the visual, biochemical and biophysical properties (which together lead to the signal observed by any remote sensing sensor) of most natural ecosystems, though the strength of these dynamics also differs amongst ecosystems. In central Europe, coniferous forests for example might appear comparably stable in their visual appearance (and therefore their spectral properties) while for example grassland ecosystems but also broadleaved forests experience drastic seasonal transformations of their visual, biochemical and biophysical properties. Farming practices add to these dynamics through harvesting and other measures. Depending on the system under consideration, fire, drought and other disturbances should play similar roles. The strong variability in reflectance over the year changes how spatial variability in reflectance is linked to species counts. Phenology, land-use and other agents may even reverse the respective sign of correlation.

Concerning the applied spectral variability measure we observed that the k-means-based measure was a lot more stable over the year than the measure based on the mean Euclidean distance. We think that this is related to the sensitivity of the Euclidean distance measure to spectrally extreme classes while the number of different reflectance spectra is disregarded. That is, while for the Euclidean distance few land-use classes showing a strong spectral difference can lead to a high mean variability (consider for example a deciduous forest area adjacent to meadows), the k-meansbased measure will return a very low number of classes (two classes in the example). As a consequence, the Euclidean distance measure is likely to change more drastically with season than the k-means-based. For example a deciduous broadleaved forest losing its foliage will still be one class in the k-means-based measure while the change from canopy-dominated reflectance to 
reflectance dominated by the forest floor is likely to affect the Euclidean distance measure notably. This temporal instability of the relation between species counts and spectral variability challenges the applicability of the SVH for a prediction of species counts with the given spatial grain of the study.

\subsection{Moving window size}

In our experiment, the size of the study area was varied by changing the size of a moving window defining the target areas of our correlation analyses. Generally, species counts correlated better with spectral variability (higher maximum correlation values) if the number of included mapping units was smaller. This result is in clear contrast to findings based on finer grains in smaller areas (Rocchini 2004, Oldeland 2010).

In our study, increasing window sizes smoothed out correlation extremes and led to less accentuated correlation patterns (Fig. 6). The results furthermore showed that for $r=6$ or more, edge effects started to dominate the observed correlation patterns. The edge effects were caused by missing mapping units in the outer parts of the moving window which conforms to a reduction of study area size in the moving window. In agreement with the general effect of window sizes, this reduction in mapping units led to an increase of the observed correlations for these areas. The larger the area of reference the higher the chance to include at the same time sub-areas with negative and positive correlations. Correlations are accordingly minimized if the entire area is subjected to a correlation analysis (see fig. 2). Weak signals for large areas have also been reported by Rocchini et al (2014) while Oindo and Skidmore (2002) found a positive link. Apart from grain effects, comparably small study sites may have contributed to the positive results reported in most of the studies listed in Table 1 because the chance of including regions with different signals is reduced. The effect of spatial grain of the remote sensing data is discussed in section 5.4. 


\subsection{Location}

Relations between MODIS-based spectral variability and plant species counts have been investigated for North America by Rocchini et al. (2014), with the result of no clear signal. Rocchini et al (2014) hypothesized that the unequal size of their mapping units was hiding the signal. In view of our results we would add spatial non-stationarity in the signal as an additional candidate explanation. Throughout the study area a heterogeneous pattern of positive, negative, low and high correlations between species counts and spectral variability was observed. As expected, high correlations were observed if a local spectral gradient matched up with a local gradient in species counts (Fig. 4 and 5). However, our results clearly demonstrated that the underlying ecological hypothesis of the SVH, that is, a higher spectral variability conforms with higher species counts due to an increased environmental heterogeneity does not always apply. We found an almost equal number of areas where the spectral variability was negatively correlated with species counts. We presented examples showing that reflectance heterogeneity at a given spatial grain is not necessarily linked to increased species counts. The reasons are multi-faceted: First, the surfaces that contribute to the heterogeneity in reflectance may be species poor (like arable fields with different crops). Second, apparently homogeneous reflectance may be heterogeneous at sub-pixel levels. In our investigation, pixels measured $0.5 \mathrm{~km}$ by $0.5 \mathrm{~km}$ and did therefore, in most of the times, include a mosaic of habitats. Third, stands with homogeneous reflectance can feature comparably high species numbers. For example, in Europe an extensively used, calcareous grassland area may host much more species (Wilson et al. 2012) than a spectrally diverse mosaic of other plant communities.

Hence, we conclude that while local applications of the SVH might be feasible if the underlying triggers are well understood, the chances of a consistent signal across broader regions are poor. In our analysis, we treated all landcover types equally. This means that habitats, like arable fields and sealed surfaces, were included that do not contribute much to overall plant species richness. 
However, mosaics including urban fabric rank among the most species rich areas in Germany regarding vascular plants (Kühn et al. 2004). This shows that masking out such landcover components could lead to inconsistencies. The Spectral Variability Hypothesis (SVH) does not make differences between habitat types.

\subsection{Spatial grain and the measure of biodiversity}

In our study, we did not investigate the effect of spatial grain on the results that are therefore valid for a certain, coarse pixel size of $0.5 \mathrm{~km}$ by $0.5 \mathrm{~km}$. It would be certainly worthwhile to test the effects of increased and reduced pixel sizes such as delivered by sensors like Landsat (30 m) or Sentinel $2(10-20 \mathrm{~m})$ or even finer grains. The effects of spatial grain (up to $30 \mathrm{~m}$ ) on the link between spectral variability and species counts has been investigated by Rocchini et al. 2007. Other examples of biodiversity studies investigating effects of spatial grain in remote sensing include the original paper by Palmer et al. (2002) and work by Mairota et al. (2015).

The coarse spatial grain used here leads to a situation where the link between species counts and spectral variability can hardly be a direct one. What we see are differences at community or landscape levels and, as discussed above, communities can be rich or poor in species. This leads us to the hypothesis that smaller pixels or larger stands of individual species may increase the chance for finding a positive relationship between spectral variability and species counts. Maybe this contributed, in addition to often small areas of investigation, to the more consistent results reported in the studies listed in table 1.

In analogy to most previous studies we used species counts as a measure of plant species richness. In our case, with coarse spatial grains, this means dealing with gamma-diversity according to Whittaker (1960). This landscape-level diversity depends on the richness of individual communities (alpha-diversity according to Whittaker) and the differences between these components of the landscape mosaic. Although some of the issues discussed above will persist, it can be hypothesized 
that these differences in the species composition of different habitats (beta-diversity according to Whittaker) can be more directly and better linked to spectral variability than the resulting higherlevel species counts. Maybe, contrasts in reflectance can be more consistently linked to contrasts in species compositions as compared to counts. We leave it to upcoming studies to test this hypothesis, which we coin here as the "community variability hypothesis". Furthermore, plant functional types (defined by plant traits rather than taxonomy, Ustin \& Gamon 2010) are more closely and more mechanistically linked to contrasts in reflectance. It is certainly worth a try to relate spectral variability to counts of and differences in plant functional types.

\subsection{Spectral variability measures and their use in multivariate analyses}

We used two measures of spectral variability, one based on Euclidean distance and one based on an unsupervised classification (k-means cluster analysis) of the image and a subsequent count of classes. The latter tended to show a closer relationship to species counts. The average contrast of habitats in terms of reflectance is not as indicative for richness as the number of habitats in an area as expressed by the number of clusters resulting from the k-means algorithm.

The Spectral Variability Hypothesis (SVH) as originally formulated by Palmer et al (2000, p. 124) states that "species richness will be positively related to any objective measure (e.g. standard deviation) of the variation in the spectral characteristics of a remotely sensed image." Land cover is the main reason why this hypothesis does not hold true across landscapes. It is clear that adding land cover or the quality of habitats as an additional predictor could greatly improve the predictions but would also deviate from the original idea of the SVH. Differences in land cover are most likely the main reason for spatial non-stationarity in the link between spectral variability and species counts. However, our study aimed at testing the hypothesis cited above and therefore did not take account of a possible use of spectral variability in multivariate analyses. For example, it might well be that adding spectral variability as a covariate in a regression analysis between land cover, climate, topography, disturbance regimes, substrates etc. and species counts leads to improved 
model fits. The fact that the SVH must be rejected for the spatial grain and area used in our study does not necessarily mean that spectral variability is useless as a predictor in other contexts.

\section{Conclusions}

This study evaluated the spectral variability hypothesis over a larger study area with a spatially continuous dataset of species counts in even-sized field mapping units, applying MODIS satellite data. Our results demonstrate obvious weaknesses in the theoretical concept of the spectral variability hypothesis which questions its general applicability. This applies to the spatial grain that has been used here but may also be relevant for other grains.

Concerning our research question, we conclude the following:

1. The spectral variability hypothesis does not hold at coarse spatial grain over larger regions in Central Europe. The observed correlations between species counts and spectral variability showed no and a marginal trend for the two spectral variability measures.

2. The performance of the spectral variability as a proxy of species numbers is influenced by the location and the extent of the reference region. We found higher correlations between species counts and spectral variability if the study areas were smaller. High spectral variability was not always connected to high species counts. We encountered many situations where comparably low spectral variation was linked to high species numbers and comparably high variability in reflectance was connected to low species counts.

3. The relation between spectral variability and species varied with season. Certain areas changed from almost no correlation to very high correlation depending on the applied scene.

4. The applied spectral variability measure has a notable influence on the observed results. In accordance with theory, the number of k-means classes within a sample plot reached higher correlations than the mean Euclidean distance. This is relevant, as earlier studies were often based 
on the latter.

We conclude that, with the investigated spatial grain, the spectral variability hypothesis does not hold. The use of measures of beta-diversity instead of counts and the use of plant functional types instead of taxonomic entities may reduce some of the problems encountered in our analysis. Others will persist, like the effects of phenology and disturbance. Although an operational use of spectral variability as a proxy in biodiversity monitoring remains a distant destination, more research along the mentioned avenues is certainly rewarding and it will advance our understanding of patterns in biodiversity.

\section{Acknowledgements}

We would like to thank the voluntary contributors to the floristic survey of Central Europe (now organized in the NetPhyD initiative) for their immense effort in assembling the species data used in this analysis. Special thanks go to the German Federal Agency for Nature Conservation (BfN), who provided the database. Duccio Rocchini and two anonymous referees provided valuable comments and helped a lot improving the manuscript. Furthermore, we are grateful to Daniel Edwin Moser, who contributed to first experiments leading to this study. Finally, we would like to thank the NASA EOSDIS Land Processes Distributed Active Archive Center (LP DAAC), USGS/Earth Resources Observation and Science (EROS) Center for providing free MODIS data. This research did not receive any specific grant from funding agencies in the public, commercial, or not-for-profit sectors.

\section{References}

Bivand, R. \& Piras, G. (2015). Comparing Implementations of Estimation Methods for Spatial Econometrics. Journal of Statistical Software, 63(18), 1-36.

Bossard, M., Feranec, J., Otahel, J. 2000: CORINE land cover technical guide - Addendum 2000. 
European Environment Agency, Technical Report No 40, Copenhagen. 105 pp.

Carlson, K.M., Asner, G.P., Hughes, R.F., Ostertag, R. \& Martin, R.E. (2007). Hyperspectral remote sensing of canopy biodiversity in Hawaiian lowland rainforests. Ecosystems, 10, 536-549.

Camathias, L., Bergamini, A., Küchler, M., Stofer, S., \& Baltensweiler, A. (2013). High-resolution remote sensing data improves models of species richness. Applied Vegetation Science, 16, 539-551.

Dungan, J., Perry, J.N., Dale, M.R.T., Legendre, P., Citron-Pousty, S., Fortin, M.-J., Jakomulska, A., Miriti, M. \& Rosenberg, M.S. (2002). A balanced view of scale in spatial statistical analysis. Ecography 25, 626-640.

Dutilleul, P. 1993. Modifying the t test for assessing the correlation between two spatial processes. Biometrics 49: 305-314.

Feilhauer, H. \& Schmidtlein, S. (2009): Mapping continuous fields of forest alpha and beta diversity. Applied Vegetation Science 12(4), 429-439.

Féret, J.-B.; Asner, G.P. (2014). Mapping tropical forest canopy diversity using high-fidelity imaging spectroscopy. Ecological Applications 24, 1289-1296.

Foody, G. \& Cutler, M. (2003). Tree biodiversity in protected and logged Bornean tropical rain forests and its measurement by satellite remote sensing. Journal of Biogeography, 30, 1053-1066.

Foody, G. \& Cutler, M. (2006). Mapping the species richness and composition of tropical forests from remotely sensed data with neural networks. Ecological Modelling, 195, 37-42.

Gillespie, T.W., Foody, G.M., Rocchini, D., Giorgi, A.P. \& Saatchi, S. (2008). Measuring and modelling biodiversity from space. Progress in Physical Geography, 32(2), 203-221.

Gould, W. (2000). Remote sensing of vegetation, plant species richness, and regional biodiversity hotspots. Ecological Applications, 10(6), 1861-1870.

Hall, K., Johansson, L.J., Sykes, M.T., Reitalu, T., Larsson, K. \& Prentice, H.C. (2010). 
Inventorying management status and plant species richness in semi-natural grasslands using high spatial resolution imagery. Applied Vegetation Science, 13, 221-233.

Hall, K., Reitalu, T., Sykes, M.T. \& Prentice, H.C. (2012). Spectral heterogeneity of QuickBird satellite data is related to fine-scale plant species spatial turnover in semi-natural grasslands. Applied Vegetation Science, 15, 145-158.

Hernández-Stefanoni, J., Dupuy, J., Johnson, K., Birdsey, R., Tun-Dzul, F., Peduzzi, A., CaamalSosa, J. P., Sánchez-Santos, G., López-Merlín, D. (2014). Improving species diversity and biomass estimates of tropical dry forests using airborne LiDAR. Remote Sensing, 6, 4741-4763.

Heumann, B.W., Hackett, R.A. \& Monfils, A.K. (2015). Testing the spectral diversity hypothesis using spectroscopy data in a simulated wetland community. Ecological Informatics, 25, 29-34. Higgins, M., Asner, G., Martin, R., Knapp, D., Anderson, C., Kennedy-Bowdoin, T., Saenz, R., Aguilar, A. \& Wright, S. (2014). Linking imaging spectroscopy and LiDAR with floristic composition and forest structure in Panama. Remote Sensing of the Environment, 154, 358-367. Hijmans, R.J. \& van Etten, J. (2012). raster: Geographic analysis and modeling with raster data. R package version 2.0-12. http://CRAN.R-project.org/package=raster

Kühn, I., Brandl, R. \& S. Klotz (2004). The flora of German cities is naturally species rich. Evolutionary Ecology Research 6: 749-764.

Laurin, G.V., Chan, J.C-W., Lindsell, J.A., Coomes, D.A., Guerriero, L., Del Frate, F., Miglietta, F. \& Valentini, R. (2014). Biodiversity mapping in a tropical West African forest with airborne hyperspectral data. PLoS ONE, 9(6), 1-10.

Levin, N., Shmida, A., Levanoni, O., Tamari, H. \& Kark, S. (2007). Predicting mountain plant richness and rarity from space using satellite-derived vegetation indices. Diversity and Distributions, 13, 692-703.

Lopatin, J., Galleguillos, M., Fassnacht, F. E., Ceballos, A., Hernández, J. (2015): Using a 
Multistructural Object-Based LiDAR Approach to Estimate Vascular Plant Richness in Mediterranean Forests With Complex Structure. IEEE Geoscience and Remote Sensing Letters, 12(5), 1008-1012.

Lopatin, J., Dolos, K., Hernández, J., Galleguillos, M., Fassnacht, F. E. (2016): Comparing Generalized Linear Models and random forest to model vascular plant species richness using LiDAR data in a natural forest in central Chile. Remote Sensing of Environment 173, pp. 200-210.

LP DAAC, 2016. https://lpdaac.usgs.gov/dataset_discovery/modis/modis_products_ta ble/mod09a1 (last access 30 July 2016).

Mahecha, M. D. \& Schmidtlein, S. (2008): Revealing biogeographical patterns by nonlinear ordinations and derived anisotropic spatial filters. Global Ecology and Biogeography 17(2), 284296.

Mairota, P., Cafarelli, B., Labadessa, R., Lovergine, F., Tarantino, C., Lucas. R.M., Nagendra, H. \& Didham, R.K. (2015): Very high resolution Earth observation features for monitoring plant and animal community structure across multiple spatial scales in protected areas. International Journal of Applied Earth Observation and Geoinformation 37: 100-105.

Meynen, E. \& J. Schmithüsen (editors: Handbuch der naturräumlichen Gliederung Deutschlands. Bundesanstalt für Landeskunde, Remagen/Bad Godesberg, 1953-1962 (9 issues in 8 books, updated map, 1:1,000,000 with major units, 1960).

Nagendra, H., Rocchini, D., Ghate, R., Sharma, B., \& Pareeth, S. (2010). Assessing plant diversity in a dry tropical forest: Comparing the utility of Landsat and IKONOS satellite images. Remote Sensing, 2, 478-496.

Netzwerk Phytodiversität Deutschland \& Bundesamt für Naturschutz (edts.) (2013): Verbreitungsatlas der Farn- und Blütenpflanzen Deutschlands. - Landwirtschaftsverlag, Münster.

Oindo, B.O. \& Skidmore, A.K. (2002). Interannual variability of NDVI and species richness in 
Kenya, International Journal of Remote Sensing, 23(2), 285-298.

Oldeland, J., Wesuls, D., Rocchini, D., Schmidt, M. \& Jürgens, N. (2010). Does using species abundance data improve estimates of species diversity from remotely sensed spectral heterogeneity? Ecological Indicators, 10, 390-396.

Palmer, M.W., Wohlgemuth, T., Earls, P., Arévalo, J.R. \& S.D. Thompson (2000): Opportunities for long-term ecological research at the Tallgrass Prairie Preserve, Oklahoma. In: Lajtha, K., Vanderbilt, K. (Eds.), Cooperation in Long Term Ecological Research in Central and Eastern Europe: Proceedings of ILTER Regional Workshop, Budapest, Hungary, 22-25 June, 1999, pp. $123-128$.

Palmer, M.W., Earls, P.G., Hoagland, B.W., White, P.S., Wohlgemuth, T. (2002). Quantitative tools for predicting species lists. Environmetrics, 13, 121-137.

Rocchini, D., Chiarucci, A. \& Loiselle, S.A. (2004). Testing the spectral variation hypothesis by using satellite multispectral images. Acta Oecologica, 26, 117-120.

Rocchini, D. (2007). Effects of spatial and spectral resolution in estimating ecosystem $\alpha$-diversity by satellite imagery. Remote Sensing of Environment, 111, 423-434.

Rocchini, D., Balkenhol, N., Carter, G.A., Foody, G.M., Gillespie, T.W., He, K.S., Kark, S., Levin, N., Lucas, K., Luoto, M., Nagendra, H., Oldeland, J., Ricotta, C., Southworth, J. \& Neteler, M. (2010). Remotely sensed spectral heterogeneity as a proxy of species diversity: Recent advances and open challenges. Ecological Informatics, 5, 318-329.

Rocchini, D., Dadalt, L., Delucchi, L., Neteler, M. \& Palmer, M.W. (2014): Disentangling the role of remotely sensed spectral heterogeneity as a proxy for North American plant species richness. Community Ecology 15: 37-43.

Salomonson, V.V., Barnes, W., Maymon, P.W., Montgomery, H.E. \& Ostrow, H. (1989). MODIS: advanced facility instrument for studies of the Earth as a system. IEEE Trans. Geoscience Remote 
Sens. 27, 145-153.

Schmidtlein, S. \& Sassin, J. (2004). Mapping of continuous floristic gradients in grassland using hyperspectral imagery. Remote Sensing of Environment, 92, 126-138.

Simonson, W., Allen, H., \& Coomes, D. (2012). Use of an airborne LiDAR system to model plant species composition and diversity of Mediterranean oak forests. Conservation Biology, 26, 840850.

Skidmore, A. K., Pettorelli, N., Coops, N.C., Geller, G.N. Hansen, M., Lucas, R., Mücher, C. A., 'Connor, B., Paganini, Pereira, H.M., Schaepman, M. E., Turner, W., Wang, T. \& Wegmann, M. (2015). Environmental science: Agree on biodiversity metrics to track from space”. Nature. 523 (7561): 403-405.

Ustin, S.L. \& Gamon, J.A. 2010: Remote sensing of plant functional types. New Phytologist 186: $795-816$.

Viedma, O. Torres, I., Pérez, B. \& Moreno, J.M. (2012). Modeling plant species richness using reflectance and texture data derived from QuickBird in a recently burned area of Central Spain. Remote Sensing of Environment, 119, 208-221.

Wang, K., Franklin, S.E., Guo, X. \& Cattet, M. (2010). Remote sensing of ecology, biodiversity and conservation: a review from the perspective of remote sensing specialists. Sensors, 10(11), 96479667.

Waser, L.T., Stofer, S., Schwarz, M., Küchler, M., Ivtis, E. \& Scheidegger, C. (2004). Prediction of biodiversity - regression of lichen species richness on remote sensing data. Community ecology, $5(1), 121-133$.

Wilson, J.B., Peet, R.K., Dengler, J., Pärtel, M. 2012. Plant species richness: the world records. Journal of Vegetation Science 23: 796-802.

Whittaker, R. H. (1960) Vegetation of the Siskiyou Mountains, Oregon and California. Ecological 
Monographs, 30, 279-338. 